\title{
Cooperative conditions for the existence of rainbow matchings
}

\author{
Ron Aharoni \\ Deparment of Mathematics \\ Technion \\ Haifa, Israel \\ and Moscow Institute of Physics and Technology (MIPT) \\ Dolgoprudny, Russia \\ raharoni@gmail.com \\ Joseph Briggs \\ Department of Mathematics and Statistics \\ Auburn University \\ AL, U.S.A. \\ jgb0059@auburn.edu

\section{Minho Cho} \\ Department of Mathematical Sciences \\ KAIST \\ Daejeon, Republic of Korea \\ mhc0925@kaist.ac.kr
}

\author{
Jinha Kim* \\ Discrete Mathematics Group \\ Institute for Basic Science (IBS) \\ Daejeon, Republic of Korea \\ jinhakim@ibs.re.kr
}

Submitted: Mar 18, 2020; Accepted: Nov 25, 2021; Published: Jan 28, 2022

(C) The authors. Released under the CC BY-ND license (International 4.0).

\begin{abstract}
Let $k>1$, and let $\mathcal{F}$ be a family of $2 n+k-3$ non-empty sets of edges in a bipartite graph. If the union of every $k$ members of $\mathcal{F}$ contains a matching of size $n$, then there exists an $\mathcal{F}$-rainbow matching of size $n$. Replacing $2 n+k-3$ by $2 n+k-2$, the result is true also for $k=1$, and it can be proved (for all $k$ ) both topologically and by a relatively simple combinatorial argument. The main effort is in gaining the last 1 , which makes the result sharp.
\end{abstract}

Mathematics Subject Classifications: 05C70, 05C35, 05D15

\footnotetext{
${ }^{*}$ Corresponding Author.
} 


\section{Introduction}

Throughout the paper, "family" means "multiset", meaning that elements may repeat. To differentiate the notation, we use round brackets for families, and (as usual) curly brackets for sets. For a family $\mathcal{F}$, we write $\mathcal{F} \backslash\{F\}$ and $\mathcal{F} \cup\{F\}$ in the family sense. That is, $\mathcal{F} \backslash\{F\}$ contains one less copy of $F$ than $\mathcal{F}$ if $F \in \mathcal{F}$, and $\mathcal{F} \cup\{F\}$ contains one more copy of $F$ than $\mathcal{F}$.

Given a family $\mathcal{S}=\left(S_{1}, \ldots, S_{m}\right)$ of sets, an $\mathcal{S}$-rainbow set is the image of a partial choice function of $\mathcal{S}$. So, it is a set $\left\{x_{i_{j}} \mid j \leqslant k\right\}$, where $1 \leqslant i_{1}<\cdots<i_{k} \leqslant m$ and $x_{i_{j}} \in S_{i_{j}}$.

A complex is a closed down hypergraph, meaning that any subset of any edge is an edge. The injectivity - at most one element from every set $S_{i}$ - is a "smallness" condition, in the sense that the set of injective choices is a complex. Hence statements of interest are of the form "there exists a large rainbow set satisfying certain conditions (like being a matching)". The classical theorem of this type is Hall's marriage theorem.

Below, again, $\mathcal{S}=\left(S_{1}, \ldots, S_{m}\right)$ is a family of sets. For a set $I \subseteq[m]$, let $\mathcal{S}_{I}=\bigcup_{i \in I} S_{i}$.

Theorem 1. If $\left|\mathcal{S}_{J}\right| \geqslant|J|$ for every $J \subseteq[m]$ then there is a full rainbow set, that is, a rainbow set of size $m$.

Another well-known rainbow result is Drisko's theorem, on rainbow matchings. The following slightly more general version of the original theorem was proved in [1]:

Theorem 2. [7] 2n-1 matchings in a bipartite graph, of size $n$ each, have a rainbow matching of size $n$.

There is a conspicuous difference between the two theorems: in the first the condition is "cooperative", namely it is on subfamilies of $\mathcal{S}$, whereas in the second it is on singletons - each $S_{i}$ is assumed to be large by itself. On the other hand, there is a condition on the number of the sets $S_{i}$.

\subsection{A cooperative version of the Kalai-Meshulam theorem}

A complex $\mathcal{C}$ is said to be $d$-Leray if $\tilde{H}_{k}(\mathcal{C}[S])=0$ for all $S \subseteq V$ and all $k \geqslant d\left(\tilde{H}_{k}\right.$ is the reduced $k$-th homology group). Let $\lambda(\mathcal{C})$ be the smallest number $d$ such that $\mathcal{C}$ is $d$-Leray.

A basic result in this direction is a theorem of Kalai and Meshulam [11]:

Theorem 3. Let $\mathcal{M}$ and $\mathcal{C}$ be a matroid and a complex, respectively, on the same ground set. If $\lambda\left(l k_{\mathcal{C}}(S)\right)<\operatorname{rank}_{\mathcal{M}}(V \backslash S)$ for every $S \in \mathcal{C}$ then $\mathcal{M} \backslash \mathcal{C} \neq \emptyset$.

Here $l k_{\mathcal{C}}(S)=\{T \subseteq V \backslash S \mid S \cup T \in \mathcal{C}\}$. The theorem above is a re-formulation of Theorem 1.6 in [11].

The following was proved in [12]:

Theorem 4. For any complex $\mathcal{C}$ and set $S \in \mathcal{C}, \lambda\left(l k_{\mathcal{C}}(S)\right) \leqslant \lambda(\mathcal{C})$.

Theorems 3 and 4, combined, yield the following: 
Theorem 5. If $\lambda(\mathcal{C}) \leqslant d$ and $\mathcal{S}=\left(S_{1}, \ldots, S_{d+k}\right)$ is a family of subsets of $V(\mathcal{C})$ satisfying $\mathcal{S}_{I} \notin \mathcal{C}$ whenever $I \subseteq[d+k]$ is of size $k$, then there exists an $\mathcal{S}$-rainbow non-C set.

Proof. By duplicating vertices, if necessary (a vertex having a distinct copy for every set $S_{i}$ it belongs to), we may assume that the sets $S_{i}$ are disjoint. Let $\mathcal{M}$ be the partition matroid defined by the sets $S_{i}$. By Theorems 4 and 3 it suffices to show that if $S \in \mathcal{C}$ then $\operatorname{rank}_{\mathcal{M}}(V \backslash S)>d$. This follows from the condition $\mathcal{S}_{I} \notin \mathcal{C} \quad(|I| \geqslant k)$ and the fact that $\operatorname{rank}_{\mathcal{M}}(A)=\left|\left\{i: A \cap S_{i} \neq \emptyset\right\}\right|$.

This is a "cooperative" version of the Kalai-Meshulam theorem, namely many sets join forces to contain a set not belonging to $\mathcal{C}$.

\subsection{A cooperative version of Theorem 2}

For a set $F$ of edges we denote by $\nu(F)$ the maximal size of a matching in $F$. For a family $\mathcal{F}=\left(F_{1}, \ldots, F_{m}\right)$ of sets of edges, we denote by $\nu_{R}(\mathcal{F})$ the maximal size of an $\mathcal{F}$-rainbow matching.

Let $t$ be an integer, and let $n \leqslant t$. Let $\mathcal{C}$ be the complex consisting of all $F \subseteq E\left(K_{t, t}\right)$, satisfying $\nu(F)<n$. In [3] it was shown that $\lambda(\mathcal{C}) \leqslant 2 n-2$. Together with Theorem 5 this yields:

Theorem 6. $2 n+k-2$ sets of edges in a bipartite graph, the union of any $k$ of which contains a matching of size $n$, have a rainbow matching of size $n$.

Notation 7 . We write $(m, k, n) \rightarrow_{\mathcal{B}} q$ for the statement "every $m$ nonempty sets of edges in a bipartite graph, the union of every $k$ of which contains a matching of size $n$, have a rainbow matching of size $q "$.

In this notation, the theorem says that $(2 n+k-2, k, n) \rightarrow_{\mathcal{B}} n$. The case $k=1$ is Theorem 2. The main result of this paper is that for $k>1$ this can be improved by 1 , thereby obtaining a sharp bound.

Theorem 8. $(2 n+k-3, k, n) \rightarrow_{\mathcal{B}} n$ whenever $1<k \leqslant n$.

The sharpness of this result, namely the fact that $(2 n+k-4, k, n) \nexists_{\mathcal{B}} n$ for any $k$, is given by the following example. In $C_{2 n}$, take the odd edges matching repeated $n-1$ times, the even edges matching repeated $n-2$ times, and a singleton set, consisting of an even edge, repeated $k-1$ times. Explicitly:

Example 9. Consider a complete bipartite graph $K_{n, n}$ with sides $\left\{a_{1}, a_{2}, \ldots, a_{n}\right\}$ and $\left\{b_{1}, b_{2}, \ldots, b_{n}\right\}$. Let

$$
S_{i}= \begin{cases}\left\{a_{1} b_{1}, a_{2} b_{2}, \ldots, a_{n} b_{n}\right\} & \text { if } i \in[n-1], \\ \left\{a_{1} b_{2}, a_{2} b_{3}, \ldots, a_{n-1} b_{n}, a_{n} b_{1}\right\} & \text { if } i \in[2 n-3] \backslash[n-1], \\ \left\{a_{1} b_{2}\right\} & \text { if } i \in[2 n+k-4] \backslash[2 n-3] .\end{cases}
$$

Let $\mathcal{S}=\left(S_{i} \mid i=1, \ldots, 2 n+k-4\right)$. Then for any $I \subseteq[2 n+k-4]$ with $|I| \geqslant k$, $\nu\left(\mathcal{S}_{I}\right) \geqslant n$, and $\nu_{R}(\mathcal{S})<n$. 
Remark 10. After our result was obtained, Holmsen and Lee [10] gave a topological proof of Theorem 8, using a strong version of Theorem 3. Their result is a somewhat stronger version of Theorem 8 .

\subsection{Cooperative versions of Colorful Caratheodory}

Part of the motivation for Theorem 8 comes from the existence of cooperative versions of a famous rainbow result - Bárány's Colorful Caratheodory theorem [6]. In fact, as we shall see below (first proof of Theorem 25), the affinity is not merely formal. Theorem 6 follows from a cooperative version of Colorful Caratheodory.

Wegner [13] noted that the complex $\mathcal{C}$ of sets of vectors in $\mathbb{R}^{d}$ not containing a given vector $v$ in their convex hull satisfies $\lambda(\mathcal{C})=d$. Similarly, the complex $\mathcal{D}$ of sets not containing $v$ in their cone (set of non-negative combinations) satisfies $\lambda(\mathcal{D})=d-1$. This, together with Theorem 5, yields:

Theorem 11. Let $v \in \mathbb{R}^{d}$.

1. If $\mathcal{S}=\left(S_{1}, \ldots, S_{d+k}\right)$ is a family of subsets of $\mathbb{R}^{d}$ such that $v \in \operatorname{conv}\left(\mathcal{S}_{K}\right)$ for every $K \subseteq[d+k]$ of size $k$, then there exists an $\mathcal{S}$-rainbow set $S$ such that $v \in \operatorname{conv}(S)$.

2. If $\mathcal{S}=\left(S_{1}, \ldots, S_{d+k-1}\right)$ is a family of subsets of $\mathbb{R}^{d}$ such that $v \in \operatorname{cone}\left(\mathcal{S}_{K}\right)$ for every $K \subseteq[d+k-1]$ of size $k$, then there exists an $\mathcal{S}$-rainbow set $S$ such that $v \in \operatorname{cone}(S)$.

The case $k=2$ of part (1) of the theorem was strengthened by Holmsen-Pach-Tverberg [9] and Arocha et.al. [5]:

Theorem 12. If $S_{1}, \ldots, S_{d+1}$ are non-empty sets in $\mathbb{R}^{d}$, and $v \in \operatorname{conv}\left(S_{i} \cup S_{j}\right)$ whenever $1 \leqslant i<j \leqslant d+1$, then there is a rainbow set $S$ with $v \in \operatorname{conv}(S)$.

Holmsen [8] gave a topological proof of this result, using a notion he called "near $d$-Lerayness", which means that $l k_{\mathcal{C}}(S)$ is $d$-Leray for every non-empty $S \in \mathcal{C}$. The same argument can be used to prove the analogous strengthening for all $k>1$ :

Theorem 13. Let $k>1$, and let $\mathcal{S}=\left(S_{1}, \ldots, S_{d+k-1}\right)$ be a family of non-empty sets in $\mathbb{R}^{d}$, such that every $k$ of them contain $v$ in the convex hull of their union. Then there is an $\mathcal{S}$-rainbow set containing $v$ in its convex hull.

The analogous strengthening of part (2) of Theorem 11 is false, as witnessed by simple counterexamples.

Example 14. Let $v_{1}, \ldots, v_{d+1}$ be the vertices of a $d$-dimensional simplex $\sigma \subseteq \mathbb{R}^{d}$ whose barycenter is the origin. Let $v$ be the barycenter of face $\left\{v_{1}, \ldots, v_{d}\right\}$ of $\sigma$. Consider the family $\mathcal{S}=\left(S_{1}, \ldots, S_{d+k-2}\right)$ of non-empty sets in $\mathbb{R}^{d}$, where $S_{i}=\left\{v_{1}, \ldots, v_{d}\right\}$ for $1 \leqslant i \leqslant d-1$ and $S_{j}=\left\{v_{d+1}\right\}$ for $d \leqslant j \leqslant d+k-2$. Among any $k$ sets in $\mathcal{S}$, at least one is $S_{i}$ for some $1 \leqslant i \leqslant d-1$, hence the convex cone spanned by their union contains $v$. However, there is no $\mathcal{S}$-rainbow set $S$ such that $v \in \operatorname{cone}(S)$. 


\section{Rainbow paths}

The proof of Theorem 8 is based on a combinatorial proof of the result $(2 n+k-2, k, n) \rightarrow_{\mathcal{B}}$ $n$, and analysis of the extreme case. This proof, in turn, uses a lemma on rainbow paths in networks. To get the extra 1 we analyze the extreme cases of that lemma. The analysis uses ideas from an analogous lemma in [4], which is the case $k=1$. But apart from a higher level of complexity, there is the difference that for $k>1$ the analysis leads to an improvement of 1 in the theorem - which was not the case for $k=1$.

A network is a triple $\mathcal{N}=(D, s, t)$, where $D$ is a digraph, and $s, t$ are two special vertices in it, called source and target. We assume that there are no edges going out of $t$ or into $s$. We write $V(\mathcal{N})$ for $V(D)$. The set $V(\mathcal{N}) \backslash\{s, t\}$ is denoted by $V^{\circ}(\mathcal{N})$, and its elements are called "inner vertices". For an $s-t$ path $P$ let $V^{\circ}(P)=V^{\circ}(\mathcal{N}) \cap V(P)$. Two $s-t$ paths $P, Q$ are said to be internally disjoint if $V^{\circ}(P) \cap V^{\circ}(Q)=\emptyset$.

For an $s-t$ path $Q$ let $B(Q)$ be the set of backward edges on $Q$, namely those directed edges $p q$ where $p, q \in V(Q)$ and $q$ precedes $p$ on $Q$. Let $s_{Q}$ be the vertex following $s$ in $Q$, and $t_{Q}$ the vertex preceding $t$ in $Q$. Define $U(Q)=\left\{v s_{Q} \mid v \in V^{\circ}(\mathcal{N}) \backslash V(Q)\right\} \cup\left\{t_{Q} u \mid u \in\right.$ $\left.V^{\circ}(\mathcal{N}) \backslash V(Q)\right\}$. (" $U$ " stands for "useless", since such edges cannot be used as shortcuts - this will be clarified below).

We shall borrow a term - "regimented" - from [4], but its use is a bit different here.

Definition 15. Let $\mathcal{F}$ be a family of sets of edges in $\mathcal{N}$. A regimentation of $\mathcal{F}$ is a pair $\mathcal{R}=(\mathcal{Q}=\mathcal{Q}(\mathcal{R}), I=I(\mathcal{R}))$, where $\mathcal{Q}$ is a set of internally disjoint $s-t$ paths, and $I$ is a function from a subset $\mathcal{E}=\mathcal{E}(\mathcal{R})$ of $\mathcal{F}$ (the "essential" sets) onto $\mathcal{Q}$, satisfying the following conditions:

1. $\bigcup_{Q \in \mathcal{Q}} V(Q)=V(\mathcal{N})$,

2. $E(I(F)) \subseteq F$ for every $F \in \mathcal{E}$, and

3. $\left|I^{-1}(Q)\right|=|E(Q)|-1$ for every $Q \in \mathcal{Q}$.

Let $\mathcal{I} \mathcal{E}(\mathcal{R})=\mathcal{F} \backslash \mathcal{E}(\mathcal{R})$ (the "inessential" sets) and $B(\mathcal{R})=\bigcup_{Q \in \mathcal{Q}} B(Q)$. If such a regimentation $\mathcal{R}$ exists, we say then that $\mathcal{F}$ is regimented by $\mathcal{R}$.

Conditions (1) and (3) imply:

Lemma 16. $|\mathcal{E}(\mathcal{R})|=\left|V^{\circ}(\mathcal{N})\right|$.

Proof. Since $\mathcal{E}(\mathcal{R})=\bigcup_{Q \in \mathcal{Q}} I^{-1}(Q)$, we have $|\mathcal{E}(\mathcal{R})|=\sum_{Q \in \mathcal{Q}}\left|I^{-1}(Q)\right|$. Then by the condition (3) of a regimentation, we have

$$
|\mathcal{E}(\mathcal{R})|=\sum_{Q \in \mathcal{Q}}\left|I^{-1}(Q)\right|=\sum_{Q \in \mathcal{Q}}(|E(Q)|-1)=\sum_{Q \in \mathcal{Q}}\left|V^{\circ}(Q)\right|
$$

Since $\mathcal{Q}$ is a set of internally disjoint $s-t$ paths, the condition (1) of a regimentation implies $\sum_{Q \in \mathcal{Q}}\left|V^{\circ}(Q)\right|=\left|V^{\circ}(\mathcal{N})\right|$, and hence we obtain $|\mathcal{E}(\mathcal{R})|=\left|V^{\circ}(\mathcal{N})\right|$. 
Notation 17 (Pruning and concatenation of paths). If $P$ is a directed path and $x \in V(P)$ then $P x$ is the part of $P$ up to and including $x$, and $x P$ is the part of $P$ starting at $x$. If two paths $P$ and $Q$ meet at a vertex $x$, then $P x Q$ denotes the walk obtained by concatenating $P x$ and $x Q$. If the endpoint of a path $P$ coincides with the initial point in a path $Q$, we write $P Q$ for the walk that is the concatenation of $P$ and $Q$.

Lemma 18. Suppose $\mathcal{F}$ is regimented by $\mathcal{R}=(\mathcal{Q}, I)$, and let $B=B(\mathcal{R}), \mathcal{I E}=\mathcal{I E}(\mathcal{R})$. If there is no $\mathcal{F}$-rainbow $s-t$ path, then $\bigcup \mathcal{I E} \subseteq B$ and $\bigcup I^{-1}(Q) \subseteq E(Q) \cup B \cup U(Q)$ for every $Q \in \mathcal{Q}$.

(For a set $\mathcal{K}$ of sets $\bigcup \mathcal{K}$ is the union of all sets in $\mathcal{K}$.)

Proof. Let $v u$ be an edge belonging to $F$ for some $F \in \mathcal{F}$. Assume that $v \in V\left(Q_{1}\right), \quad u \in$ $V\left(Q_{2}\right)$. Let $P=Q_{1} v u Q_{2}$ (see Notation 17 ).

To obtain the conclusion of the lemma, we will show the following.

1. When $Q_{1}=Q_{2}, P$ is an $\mathcal{F}$-rainbow $s-t$ path unless $v u \in B\left(Q_{1}\right)$ or $v u \in E\left(Q_{1}\right)$ and $F \in I^{-1}\left(Q_{1}\right)$.

2. When $Q_{1} \neq Q_{2}, P$ is an $\mathcal{F}$-rainbow $s-t$ path unless $v=t_{Q_{1}}$ and $F \in I^{-1}\left(Q_{1}\right)$, or $u=s_{Q_{2}}$ and $F \in I^{-1}\left(Q_{2}\right)$.

First suppose that $Q_{1}=Q_{2}$. If $v$ precedes $u$ on $Q_{1}$ and $v u \notin E\left(Q_{1}\right)$, then $P$ is an $\mathcal{F}$-rainbow $s-t$ path, since by part (3) of Definition 15 it has enough represented sets for its length. If $v u \in E\left(Q_{1}\right)$, then $P$ is an $\mathcal{F}$-rainbow $s-t$ path unless $F \in I^{-1}\left(Q_{1}\right)$. This proves (1).

Now assume $Q_{1} \neq Q_{2}$. We may assume that $v \in V^{\circ}\left(Q_{1}\right)$ and $u \in V^{\circ}\left(Q_{2}\right)$ since if not the claim is a special case of (1). Then $Q_{1} v$ and $u Q_{2}$ are rainbow, and they have enough represented sets in $I^{-1}\left(Q_{1}\right)$ and $I^{-1}\left(Q_{2}\right)$, respectively. If $F \notin I^{-1}\left(Q_{1}\right) \cup I^{-1}\left(Q_{2}\right)$, then $P$ is rainbow. If $F \in I^{-1}\left(Q_{1}\right)$ and $v \neq t_{Q_{1}}$, then $Q_{1} v u$ is rainbow since it has enough represented sets in $I^{-1}\left(Q_{1}\right)$, since it has length at most $\left|E\left(Q_{1}\right)\right|-1$. Similarly if $F \in I^{-1}\left(Q_{2}\right)$ and $u \neq s_{Q_{2}}$, then $v u Q_{2}$ is rainbow since it has enough represented sets in $I^{-1}\left(Q_{2}\right)$. In both cases $P$ is rainbow, which proves $(2)$.

Since we assume there is no $\mathcal{F}$-rainbow $s-t$ path, if $F \in \mathcal{I E}$, then $v u \in B$ by (1) and (2). Thus $\bigcup \mathcal{I E} \subseteq B$. If $F \in I^{-1}(Q)$ for some $Q \in \mathcal{Q}$, then $v u \in E(Q) \cup B \cup U(Q)$ by (1) and (2). Thus $\cup I^{-1}(Q) \subseteq E(Q) \cup B \cup U(Q)$.

Corollary 19. Let $\mathcal{F}$ be regimented by $\mathcal{R}$, and assume that there is no $\mathcal{F}$-rainbow $s-t$ path. If $F \in \mathcal{I E}(\mathcal{R})$ then $F$ does not contain an $s-t$ path.

In fact, $F$ does not even contain an edge $s y$.

Lemma 20. Let $P, Q$ be $s-t$ paths in a network $(D, s, t)$. If $E(P) \subseteq E(Q) \cup B(Q) \cup$ $\tilde{B} \cup U(Q)$ for some collection $\tilde{B}$ of edges that are vertex-disjoint from $Q$, then $P=Q$. 
Proof. The only edge leaving $s$ in $E(Q) \cup B(Q) \cup \tilde{B} \cup U(Q)$ is $s s_{Q} \in E(Q)$, and the only edge to $t$ is $t_{Q} t \in E(Q)$. So these are necessarily the first and last edges of $P$. Therefore $P$ has no edges from $U(Q)$, since the in-degree of $s_{Q}$ and the out-degree of $t_{Q}$ in $P$ are 1 .

As $E(Q) \cup B(Q)$ and $\tilde{B}$ are disconnected, $E(P) \cap \tilde{B}=\emptyset$. It remains to show that $E(P) \cap B(Q)=\emptyset$, which follows from the fact that $P$ does not repeat vertices.

Combining Lemmas 18 and 20 yields:

Corollary 21. Let $\mathcal{F}$ be regimented by $\mathcal{R}$, and having no rainbow $s-t$ path. If $F \in \mathcal{E}(\mathcal{R})$ then $I(F)$ is the only $s-t$ path contained in $F$.

By Corollaries 19 and 21, we can obtain the following corollary.

Corollary 22. Let $\mathcal{F}$ be regimented by $\mathcal{R}$, and having no rainbow $s-t$ path. Then $F \in \mathcal{E}(\mathcal{R})$ if and only if $F$ contains an $s-t$ path, and equivalently, $F \in \mathcal{I E}(\mathcal{R})$ if and only if $F$ does not contain an $s-t$ path.

The following argument will be used twice, and hence it receives separate mention:

Lemma 23. Let $\mathcal{G}, \mathcal{H}$ be two families of sets of edges, none of which possesses a rainbow $s-t$ path. Suppose that $\mathcal{G}$ is regimented by $\mathcal{R}=(\mathcal{Q}, I)$ and $\mathcal{H}$ is regimented by $\mathcal{S}=(\mathcal{P}, J)$. Suppose that $\mathcal{G} \backslash \mathcal{H}$ consists of a single set of edges $G$, and $\mathcal{H} \backslash \mathcal{G}$ consists of single set of edges $H$. Then either $G \in \mathcal{I E}(\mathcal{R})$ and $H \in \mathcal{I E}(\mathcal{S})$, or $I(G)=J(H)$.

Proof. Let $\mathcal{K}=\mathcal{G} \cap \mathcal{H}$. So $\mathcal{G}=\mathcal{K} \cup\{G\}, \mathcal{H}=\mathcal{K} \cup\{H\}$.

By Corollary 22, it is obvious that

$$
\mathcal{K} \cap \mathcal{E}(\mathcal{R})=\mathcal{K} \cap \mathcal{E}(\mathcal{S})
$$

By Corollary 21, $I(K)=J(K)$ for every $K \in \mathcal{K} \cap \mathcal{E}(\mathcal{R})$. Hence

$$
\bigcup_{K \in \mathcal{E}(\mathcal{R}) \backslash\{G\}} V(I(K))=\bigcup_{K \in \mathcal{E}(\mathcal{S}) \backslash\{H\}} V(J(K))
$$

Let us first show that $G \in \mathcal{I E}(\mathcal{R})$ if and only if $H \in \mathcal{I E}(\mathcal{S})$. Suppose that $G \in \mathcal{I E}(\mathcal{R})$. Then $\mathcal{E}(\mathcal{R}) \subseteq \mathcal{K}$. By $(1)$ and Lemma 16 , it follows that $\mathcal{E}(\mathcal{S})=\mathcal{E}(\mathcal{R})$, so $H \in \mathcal{I E}(\mathcal{S})$. The converse implication is the same.

Assume next that $G \in \mathcal{E}(\mathcal{R})$ and $H \in \mathcal{E}(\mathcal{S})$. Let $Q_{0}=I(G)$. Consider first the case that $V^{\circ}\left(Q_{0}\right)$ consists of a single vertex $v$. We have $\bigcup_{K \in \mathcal{E}(R) \backslash\{G\}} V(I(K))=V^{\circ} \backslash\{v\}$, and hence by (2) we have also $\bigcup_{K \in \mathcal{E}(\mathcal{S}) \backslash\{H\}} V(J(K))=V^{\circ} \backslash\{v\}$. Since the interiors of paths in $\mathcal{P}$ partition $V^{\circ}$, it follows that $J(H)$ is the path svt, namely $Q_{0}$.

It remains to consider the case $\left|V^{\circ}\left(Q_{0}\right)\right|>1$. Then, not counting multiplicities, $\mathcal{P}=\mathcal{Q}$, because every path of $\mathcal{Q}$ appears as $J(K)$ for some $K \in \mathcal{K}$. The only path in $\mathcal{P}$ not covered enough times by paths $J(K), K \in \mathcal{E}(\mathcal{S}) \backslash\{H\}$, is $Q_{0}$. So, necessarily $J(H)=Q_{0}$.

The next theorem is the main step towards the proof of Theorem 8 . 
Theorem 24. Let $\mathcal{N}=(D, s, t)$ be a network with $n$ inner vertices. Let $\mathcal{F}$ be a family of $n+k-1$ sets of edges in $\mathcal{N}$, satisfying the condition that $\bigcup \mathcal{K}$ contains an $s-t$ path, for every $\mathcal{K} \subseteq \mathcal{F}$ of size $k$. Then either there exists an $\mathcal{F}$-rainbow $s-t$ path, or $\mathcal{F}$ is regimented.

The case $k=1$ of the theorem is Theorem 3.3 in [4].

It is worth noting that the weaker result, with $\mathcal{F}$ being of size $n+k$, is not hard. First, the statement:

Theorem 25. Let $\mathcal{N}=(D, s, t)$ be a network with $n$ inner vertices. Let $\mathcal{F}$ be a family of $n+k$ sets of edges in $\mathcal{N}$, satisfying the condition that $\bigcup \mathcal{K}$ contains an $s-t$ path for every $\mathcal{K} \subseteq \mathcal{F}$ of size $k$. Then there exists an $\mathcal{F}$-rainbow $s-t$ path.

Here are two proofs:

Proof 1. Observe that a set $H$ of edges in $\mathcal{N}$ contains an $s-t$ path if and only if the cone of $\left\{\chi_{b}-\chi_{a} \mid a b \in H\right\}$ contains the vector $\chi_{t}-\chi_{s}$ (here $\chi_{v}$ is the function that is 1 on $v$ and 0 on all other vertices). Also note that all these vectors reside in an $n+1$-dimensional space (they are of length $n+2$, but all are perpendicular to the all-1 vector). Apply now Theorem 11, part (2).

Proof 2. Take a maximal $\mathcal{F}$-rainbow tree $T$ rooted at $s$. Assume, for contradiction, that it does not reach $t$. Then it represents at most $n$ members of $\mathcal{F}$. Hence there are $k$ sets $F \in \mathcal{F}$ not represented in $T$. By assumption, their union contains an $s-t$ path. The first edge leaving $T$ can then be added to $T$ to yield a larger rainbow tree, which contradicts the maximality of $T$.

Definition 26 (contracting an edge $\boldsymbol{s} \boldsymbol{x}$ ). Let $s x$ be an edge of $\mathcal{N}$. We can contract $s x$ to a newly defined vertex $s^{\prime}$, that will serve as the source of a new network $\mathcal{N}^{\prime}$. Here is what this does to sets of edges, and to paths.

1. Let $F$ be a set of edges in a network $\mathcal{N}=(D, s, t)$, and let $s x$ be an edge, where $x$ is an inner vertex. The contracted set of edges $\left.F\right|_{s x \rightarrow s^{\prime}}$ is obtained from $F$ by replacing every edge $s y$ or $x y$ belonging to $F$ by the edge $s^{\prime} y$, and removing all edges $y x$.

2. An $s-t$ path $P$ is transformed by the contraction of $s x$ to an $s^{\prime}-t$ path $P^{\prime}$, defined as follows. If $x \notin V(P)$ then $P^{\prime}=P$ with $s^{\prime}$ replacing $s$. If $x \in V(P)$ then $P^{\prime}=s^{\prime} y P$ where $y$ is the vertex following $x$ in $P$ (so, the vertices in $P x$ disappear.) We also write $P^{\prime}=\left.P\right|_{s x \rightarrow s^{\prime}}$. Note that in this definition $E\left(P^{\prime}\right)$ is not necessarily equal to $\left.E(P)\right|_{s x \rightarrow s^{\prime}}$.

Proof of Theorem 24. By induction on $n$. The case $n=0$ is easy. So let $n \geqslant 1$ and assume that the theorem is valid when $n-1$ replaces $n$.

Since $n+k-1 \geqslant k, \bigcup \mathcal{F}$ contains an $s-t$ path. So there exists at least one set $G \in \mathcal{F}$ containing an edge $s x$. If $x=t$ then the path $s t$ is rainbow, so we may assume that $x \neq t$. Now contract $s x$ : for each $F \in \mathcal{F}$ let $F^{\prime}=\left.F\right|_{s x \rightarrow s^{\prime}}$. Let $\mathcal{K}^{\prime}=\left(F^{\prime} \mid F \in \mathcal{F}\right)$ for $\mathcal{K} \subseteq \mathcal{F}$. Let $\mathcal{N}^{\prime}$ be the network with vertex set $V(\mathcal{N}) \backslash\{s, x\} \cup\left\{s^{\prime}\right\}$, source $s^{\prime}$, target $t$, and edge set $\bigcup\left(\mathcal{F}^{\prime} \backslash\left\{G^{\prime}\right\}\right)$. 
Every $\mathcal{K} \subseteq \mathcal{F}$ of size $k$ contains in its union the edge set of an $s-t$ path in $\mathcal{N}$, which is easily seen to imply the same, with $s^{\prime}$ replacing $s$, for $\mathcal{K}^{\prime}$ in $\mathcal{N}^{\prime}$. By the induction hypothesis, either there exists an $\mathcal{F}^{\prime} \backslash\left\{G^{\prime}\right\}$-rainbow $s^{\prime}-t$ path $P^{\prime}$, or $\mathcal{F}^{\prime} \backslash\left\{G^{\prime}\right\}$ is regimented. In the first case, let $y$ be the vertex following $s^{\prime}$ in $P^{\prime}$. Then either $s y P^{\prime}$ or $s x y P^{\prime}$ is a rainbow $s-t$ path in $\mathcal{N}$, and we are done. So, we may assume the second possibility. Let $\mathcal{R}^{\prime}=\left(\mathcal{Q}^{\prime}, I^{\prime}\right)$ be a regimentation of $\mathcal{F}^{\prime} \backslash\left\{G^{\prime}\right\}$, and let $\mathcal{E}^{\prime}=\mathcal{E}\left(\mathcal{R}^{\prime}\right), \mathcal{I E}^{\prime}=\mathcal{I} \mathcal{E}\left(\mathcal{R}^{\prime}\right)$.

Let $\tilde{\mathcal{I E}}=\left(F \in \mathcal{F} \backslash\{G\} \mid F^{\prime} \in \mathcal{I} \mathcal{E}^{\prime}\right)$ and $\tilde{\mathcal{E}}=\left(F \in \mathcal{F} \backslash\{G\} \mid F^{\prime} \in \mathcal{E}^{\prime}\right)$.

By Lemma $16\left|\mathcal{E}^{\prime}\right|=n-1$, so

$$
|\tilde{\mathcal{I E}}|=\left|\mathcal{I} \mathcal{E}^{\prime}\right|=k-1
$$

In all claims below we assume that there is no $\mathcal{F}$-rainbow $s-t$ path.

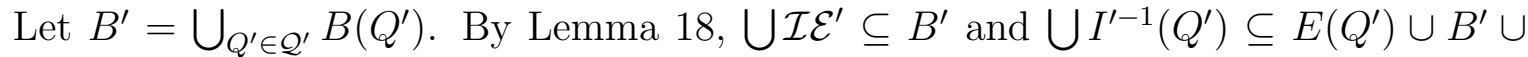
$U\left(Q^{\prime}\right)$ for every $Q^{\prime} \in \mathcal{Q}^{\prime}$.

Notation 27 (two ways of un-contracting $s x$ ). Given an $s^{\prime}-t$ path $Q^{\prime}$ in $\mathcal{N}^{\prime}$, let $Q^{\prime(1)}$ be the path obtained from $Q^{\prime}$ by replacing $s^{\prime}$ with $s$ and $Q^{\prime(2)}$ the path obtained from $Q^{\prime}$ by expanding its first edge $s^{\prime} y$ to the path $s x y$.

Our aim is to glean from $\mathcal{R}^{\prime}$ a regimentation $\mathcal{R}=(\mathcal{Q}, I)$ of $\mathcal{F}$. The set $\mathcal{E}(\mathcal{R})$ will contain $G$ and $\mathcal{Q}$ will contain $s-t$ paths $f\left(Q^{\prime}\right), \quad Q^{\prime} \in \mathcal{Q}^{\prime}$, where $f$ is an injective function defined as follows. Let $Q^{\prime} \in \mathcal{Q}^{\prime}$ and let $F \in \mathcal{F} \backslash\{G\}$ be such that $I^{\prime}\left(F^{\prime}\right)=Q^{\prime}$. By (3) and the condition of the theorem, the set $F \cup \cup \tilde{\mathcal{I E}}$ contains an $s-t$ path $Q$. Let $f\left(Q^{\prime}\right)=Q$.

Claim 28. $Q^{\prime}=\left.Q\right|_{s x \rightarrow s^{\prime}}$.

Proof. By the choice of $Q$, we have $E\left(\left.Q\right|_{s x \rightarrow s^{\prime}}\right) \subseteq F^{\prime} \cup \cup \mathcal{I} \mathcal{E}^{\prime}$. By Lemma 18, we have $F^{\prime} \cup \bigcup \mathcal{I} \mathcal{E}^{\prime} \subseteq E\left(Q^{\prime}\right) \cup B^{\prime} \cup U\left(Q^{\prime}\right)=E\left(Q^{\prime}\right) \cup B\left(Q^{\prime}\right) \cup \bigcup_{T^{\prime} \in \mathcal{Q}^{\prime} \backslash\left\{Q^{\prime}\right\}} B\left(T^{\prime}\right) \cup U\left(Q^{\prime}\right)$. The claim now follows by Lemma 20.

There are two possibilities:

(a) $x \notin V(Q)$. In this case $Q=Q^{\prime(1)}$.

(b) $x \in V(Q)$. Suppose, in this case, that $Q x$ contains inner vertices. Let $y$ be the first inner vertex of $Q x$. Then $y \in V^{\circ}\left(T^{\prime}\right)$ for some $T^{\prime} \in \mathcal{Q}^{\prime} \backslash\left\{Q^{\prime}\right\}$, and then syT is a rainbow $s-t$ path in $\mathcal{N}$ since it has enough represented sets in $I^{\prime-1}\left(T^{\prime}\right) \cup\{G\}$. So, we may assume that $V^{\circ}(Q x)=\emptyset$, meaning that the first edge on $Q$ is $s x$, meaning in turn that $Q=Q^{\prime(2)}$.

Claim 29. $s x \notin \bigcup \tilde{\mathcal{I E}}$.

Proof. Let $F_{0} \in \tilde{\mathcal{I E}}$ and suppose that $s x \in F_{0}$. Recall that $\mathcal{F}^{\prime}$ is the family of sets of edges obtained, where, for every $F \in \mathcal{F}, F^{\prime}$ is the image of $F$ under the contraction of $s x$. By the same argument as above, $\mathcal{F}^{\prime} \backslash\left\{F_{0}^{\prime}\right\}$ is regimented in $\mathcal{N}^{\prime}$, by a regimentation $\mathcal{T}=(\mathcal{Q}(\mathcal{T}), J)$. Then $G^{\prime} \in \mathcal{I E}(\mathcal{T})$ by Lemma 23 , and hence $G$ do not contain an edge yt. But this would imply that $G \cup \tilde{\mathcal{I}} \mathcal{E}(\mathcal{R})$ does not contain such an edge, and hence that it does not contain an $s-t$ path, contrary to the assumption of the theorem. 
Since $E(Q) \subseteq F \cup \cup \tilde{\mathcal{I E}}$ and $\bigcup \mathcal{I} \mathcal{E}^{\prime} \subseteq B^{\prime}$ by Lemma 18, a corollary of Claim 29 is:

$$
E(Q) \subseteq F
$$

Claim 30. The choice of $f\left(Q^{\prime}\right)$ is independent of the choice of $F$.

Proof. We have to show that if $F_{1}, F_{2} \in \mathcal{F} \backslash\{G\}$ satisfy $I^{\prime}\left(F_{i}^{\prime}\right)=Q^{\prime}, \quad i=1,2$ and $Q_{i}$ are $s-t$ paths whose edge sets are contained in $F_{i} \cup \tilde{\mathcal{I E}}(i=1,2)$ then $Q_{1}=Q_{2}$. We know that $Q_{i}$ are either $Q^{\prime(1)}$ or $Q^{\prime(2)}$. Assume, for contradiction, that $Q_{1} \neq Q_{2}$, say $Q_{1}=Q^{\prime(1)}$ and $Q_{2}=Q^{\prime(2)}$. Then $s x \in E\left(Q_{2}\right)$ and hence $s x \in F_{2}$. The set $\mathcal{F}^{\prime} \backslash\left\{F_{2}^{\prime}\right\}$ lives in $\mathcal{N}^{\prime}$, and repeating the previous argument we deduce that it has a regimentation $\mathcal{S}=(\mathcal{Q}(\mathcal{S}), J)$. By Lemma $23 J\left(G^{\prime}\right)=I^{\prime}\left(F_{2}^{\prime}\right)=Q^{\prime}$. In particular $G^{\prime} \supseteq E\left(Q^{\prime}\right)$. Since $Q_{1}=Q^{\prime(1)}$, the edge $s s_{Q^{\prime}}$ belongs to $E\left(Q_{1}\right) \subseteq F_{1}$. Then, using an edge from $G$ and edges from the sets $F \in \mathcal{F}$ such that $F^{\prime} \in I^{\prime-1}\left(Q^{\prime}\right)$ shows that $s s_{Q^{\prime}} Q^{\prime}=Q^{\prime(1)}$ is an $\mathcal{F}$-rainbow $s-t$ path (note that edges in $E\left(s_{Q^{\prime}} Q^{\prime}\right)$ are also edges of $\left.F\right)$. This is the desired contradiction.

\section{Claim 31.}

1. If $f\left(Q^{\prime}\right)=Q^{\prime(2)}$ then $G \supseteq E\left(f\left(Q^{\prime}\right)\right)$.

2. At most one $Q^{\prime} \in \mathcal{Q}^{\prime}$ satisfies $f\left(Q^{\prime}\right)=Q^{\prime(2)}$.

3. If $f\left(Q^{\prime}\right)=Q^{\prime(1)}$ for all $Q^{\prime} \in \mathcal{Q}^{\prime}$ then $G$ contains the edges of the $s-t$ path sxt.

Proof. To prove (1), let $f\left(Q^{\prime}\right)=Q^{\prime(2)}$ for some $Q^{\prime} \in \mathcal{Q}^{\prime}$.

Then, by Claim 30, sx $\in F$ for every $F^{\prime} \in I^{\prime-1}\left(Q^{\prime}\right)$. We use the same trick as in the proof of Claim 30, interchanging the roles of $F$ and $G$. Consider $\mathcal{F}^{\prime} \backslash\left\{F^{\prime}\right\}$. As above, we may assume that $\mathcal{F}^{\prime} \backslash\left\{F^{\prime}\right\}$ is regimented, by a regimentation $\left(\mathcal{P}^{\prime}, J^{\prime}\right)$. By Lemma 23, $J^{\prime}\left(G^{\prime}\right)=I^{\prime}\left(F^{\prime}\right)=Q^{\prime}$, implying that $G^{\prime} \supseteq E\left(Q^{\prime}\right)$. Then $G$ contains either $E\left(Q^{\prime(1)}\right)$ or $E\left(Q^{\prime(2)}\right)$. If $G$ contains $E\left(Q^{\prime(1)}\right)$, then $s s_{Q^{\prime}} Q^{\prime}$ (which is just $Q^{\prime(1)}$ ) is an $\mathcal{F}$-rainbow $s-t$ path: the edge $s s_{Q^{\prime}}$ represents $G$; since $\left|I^{\prime-1}\left(Q^{\prime}\right)\right|=\left|E\left(Q^{\prime}\right)\right|-1$, the other edges have enough represented sets $F \in \mathcal{F}$ such that $F^{\prime} \in I^{\prime-1}\left(Q^{\prime}\right)$ (remember that $G \notin I^{\prime-1}\left(Q^{\prime}\right)$ ). We have thus shown that $G$ does not contain $E\left(Q^{\prime(1)}\right)$, so it contains $E\left(Q^{\prime(2)}\right)$, namely $G \supseteq E\left(f\left(Q^{\prime}\right)\right)$.

Next we prove (2). Let $f\left(Q^{\prime}\right)=Q^{\prime(2)}$ for some $Q^{\prime} \in \mathcal{Q}^{\prime}$. By the above argument and Corollary 21, $J^{\prime}\left(G^{\prime}\right)=Q^{\prime}$ is the only path contained in $G^{\prime}$. This directly implies (2).

Finally, we prove (3). Assume that $f\left(Q^{\prime}\right)=Q^{\prime(1)}$ for all $Q^{\prime} \in \mathcal{Q}^{\prime}$. Let $\tilde{\mathcal{N}}$ be the network obtained from $\mathcal{N}$ by deleting the vertex $x$, and let $\tilde{F}$ be the set of edges of $\tilde{\mathcal{N}}$, obtained from $F$ by deleting all edges incident with $x$. Let $\tilde{\mathcal{Q}}=\left\{Q^{\prime(1)} \mid Q^{\prime} \in \mathcal{Q}^{\prime}\right\}$, and $\tilde{I}(\tilde{F})=f\left(I^{\prime}\left(F^{\prime}\right)\right)$. By (4) and the assumption that $f\left(Q^{\prime}\right)=Q^{\prime(1)}$ for all $Q^{\prime} \in \mathcal{Q}^{\prime}$ the set $\tilde{\mathcal{F}}=(\tilde{F} \mid F \in \mathcal{F})$ is regimented by $(\tilde{\mathcal{Q}}, \tilde{I})$. The fact that there is no $\mathcal{F}$-rainbow $s-t$ path implies that there is also no $\tilde{\mathcal{F}}_{\text {-rainbow }} s-t$ path. Therefore, by Lemma 18 , we have $\tilde{G} \cup \bigcup_{F \in \tilde{\mathcal{I}} \mathcal{E}} \tilde{F} \subseteq \bigcup_{Q \in \tilde{\mathcal{Q}}} B(Q)$. Thus

$$
G \cup \bigcup \tilde{\mathcal{I E}} \subseteq\{s x, x t\} \cup \bigcup_{Q^{\prime} \in \mathcal{Q}^{\prime}} B\left(Q^{\prime(1)}\right) \cup U(s x t) .
$$


By the assumption of the theorem, $G \cup \bigcup \tilde{\mathcal{I E}}$ contains an $s-t$ path, say $Q_{G}$. By Lemma 20 we have $Q_{G}=s x t$, and by Claim 29 we obtain $G \supseteq E\left(Q_{G}\right)$. This concludes the proof of the claim.

Remark 32. By the claim the paths $f\left(Q^{\prime}\right), \quad Q^{\prime} \in \mathcal{Q}^{\prime}$ are internally disjoint. In particular, there is at most one path $f\left(Q^{\prime}\right)$ containing $x$.

We can now complete the induction step in the proof of Theorem 24, by showing that $\mathcal{F}$ is regimented.

Case I: $\quad f\left(Q^{\prime}\right)=Q^{\prime(1)}$ for all $Q^{\prime} \in \mathcal{Q}^{\prime}$.

Let $\mathcal{Q}=\left\{f\left(Q^{\prime}\right) \mid Q^{\prime} \in \mathcal{Q}^{\prime}\right\} \cup\left\{Q_{0}\right\}$ where $Q_{0}=$ sxt. Let $\mathcal{E}=\left(F \mid F^{\prime} \in \mathcal{E}\left(\mathcal{R}^{\prime}\right)\right) \cup\{G\}$. Define $I: \mathcal{E} \rightarrow \mathcal{Q}$ by $I(F)=f\left(I^{\prime}\left(F^{\prime}\right)\right)$ for $F \neq G$, and $I(G)=Q_{0}$.

Claim 33. $(\mathcal{Q}, I)$ is a regimentation of $\mathcal{F}$.

By Remark 32 and the fact that $x \notin \bigcup_{Q^{\prime} \in \mathcal{Q}^{\prime}} V\left(f\left(Q^{\prime}\right)\right), \mathcal{Q}$ is a set of internally disjoint $s-t$ paths.

By (4) $E(I(F)) \subseteq F$ for all $F \in \mathcal{E} \backslash\{G\}$, and by part (3) of Claim $31 E(I(G))=$ $E\left(Q_{0}\right) \subseteq G$. This implies condition (2) in Definition 15 .

In addition,

$$
\left|I^{-1}(Q)\right|=\left|I^{\prime-1}\left(f^{-1}(Q)\right)\right|=\left|E\left(f^{-1}(Q)\right)\right|-1=\left|E\left(f^{-1}(Q)^{(1)}\right)\right|-1=|E(Q)|-1
$$

for all $Q \in \mathcal{Q} \backslash\left\{Q_{0}\right\}$, and

$$
\left|I^{-1}\left(Q_{0}\right)\right|=1=\left|E\left(Q_{0}\right)\right|-1 .
$$

This yields condition (3) of Definition 15 .

Furthermore, since $\bigcup_{Q^{\prime} \in \mathcal{Q}^{\prime}} V^{\circ}\left(Q^{\prime}\right)=V^{\circ}(\mathcal{N}) \backslash\{x\}$ and $V^{\circ}\left(Q^{\prime(1)}\right)=V^{\circ}\left(Q^{\prime}\right)$, we have

$$
\bigcup_{Q \in \mathcal{Q}} V^{\circ}(Q)=\bigcup_{Q^{\prime} \in \mathcal{Q}^{\prime}} V^{\circ}\left(Q^{\prime(1)}\right) \cup\{x\}=V^{\circ}(\mathcal{N}) \text {. }
$$

This implies condition (1) of Definition 15, thus completing the proof of the claim.

Case II: $f\left(Q_{0}^{\prime}\right)=Q_{0}^{\prime(2)}$ for some $Q_{0}^{\prime} \in \mathcal{Q}$.

Let $\mathcal{Q}=\left\{f\left(Q^{\prime}\right) \mid Q^{\prime} \in \mathcal{Q}^{\prime}\right\}$ and $\mathcal{E}=\left(F \mid F^{\prime} \in \mathcal{E}\left(\mathcal{R}^{\prime}\right)\right) \cup\{G\}$. Define $I: \mathcal{E} \rightarrow \mathcal{Q}$ by $I(F)=f\left(I^{\prime}\left(F^{\prime}\right)\right)$ for all $F \in \mathcal{F} \backslash\{G\}$ and $I(G)=f\left(Q_{0}^{\prime}\right)$.

Claim 34. $(\mathcal{Q}, I)$ is (here, too) a regimentation of $\mathcal{F}$.

By Remark $32, \mathcal{Q}$ is a set of internally disjoint $s-t$ paths.

By (4) $E(I(F)) \subseteq F$ for $F \in \mathcal{E} \backslash\{G\}$, and by (1) of Claim $31 E(I(G))=E\left(f\left(Q_{0}^{\prime}\right)\right) \subseteq G$, so condition (2) of Definition 15 is fulfilled.

In addition,

$$
\left|I^{-1}(Q)\right|=\left|I^{\prime-1}\left(f^{-1}(Q)\right)\right|=\left|E\left(f^{-1}(Q)\right)\right|-1=\left|E\left(f^{-1}(Q)^{(1)}\right)\right|-1=|E(Q)|-1
$$


for all $Q \neq f\left(Q_{0}^{\prime}\right)$. On the other hand, for $Q=f\left(Q_{0}^{\prime}\right)$,

$$
\left|I^{-1}(Q)\right|=\left|I^{-1}\left(f^{-1}(Q)\right)\right|+1=\left|E\left(f^{-1}(Q)\right)\right|=\left|E\left(f^{-1}(Q)^{(2)}\right)\right|-1=|E(Q)|-1 .
$$

This proves condition (3) in Definition 15.

Furthermore, since $\bigcup_{Q^{\prime} \in \mathcal{Q}^{\prime}} V^{\circ}\left(Q^{\prime}\right)=V^{\circ}(\mathcal{N}) \backslash\{x\}, V^{\circ}\left(Q^{\prime(1)}\right)=V^{\circ}\left(Q^{\prime}\right)$ and $V^{\circ}\left(Q^{\prime(2)}\right)=$ $V^{\circ}\left(Q^{\prime}\right) \cup\{x\}$, we have

$$
\bigcup_{Q \in \mathcal{Q}} V^{\circ}(Q)=\bigcup_{Q^{\prime} \in \mathcal{Q}^{\prime} \backslash\left\{Q_{0}^{\prime}\right\}} V^{\circ}\left(Q^{\prime(1)}\right) \cup V^{\circ}\left(Q_{0}^{\prime(2)}\right)=V^{\circ}(\mathcal{N}) .
$$

So, condition (1) of Definition 15 is also valid, completing the proof of the theorem.

\section{Proof of Theorem 8}

Let us first state the theorem in a slightly stronger form, that allows some of the edge sets to be empty.

Theorem 35. Let $\mathcal{S}$ be a family of $2 n+k-3$ sets of edges in a bipartite graph $G$, at most $k-2$ of them being empty. If $\nu(\bigcup \mathcal{K}) \geqslant n$ for every $\mathcal{K} \subseteq \mathcal{S}$ of size $k$ then $\nu_{R}(\mathcal{S}) \geqslant n$.

Before proving the theorem, we need the following definition.

Definition 36. For a matching $N$ in a graph, a path is called $N$-alternating if every other edge in it belongs to $N$ and it is called augmenting if its starting edge and ending edge are not in $N$.

Proof. Suppose, for contradiction, that $\nu_{R}(\mathcal{S})=: m<n$. Let $M=\left\{f_{S} \mid S \in \mathcal{S}_{0}\right\}$ be a maximal size $\mathcal{S}$-rainbow matching, where $f_{S} \in S$. Let $\mathcal{S}_{0}^{c}=\mathcal{S} \backslash \mathcal{S}_{0}$.

Let $A, B$ be the two sides of $G$. For every $h \in E(G)$ let $h_{A}$ be the $A$-vertex of $h$, and $h_{B}$ the $B$ vertex.

We construct a network $\mathcal{N}$, having the property that its paths correspond to $M$ alternating paths, and its source-target paths correspond to augmenting $M$-alternating paths. Let $V(\mathcal{N})=M \cup\{s, t\}$, where $s$ represents $U_{A}:=A \backslash \bigcup M$, and $t$ represents $U_{B}:=B \backslash \bigcup M$

To every edge $h=a b \in E(G) \backslash M(a \in A, b \in B)$ we assign an edge $F(h)$ of $\mathcal{N}$, as follows.

1. If $a \in f \in M, b \in g \in M$ then $F(h)=f g$.

2. If $a \in U_{A}$ and $b \in g \in M$ then $F(h)=s g$.

3. If $b \in U_{B}$ and $a \in f \in M$ then $F(h)=f t$.

4. If $a \in U_{A}$ and $b \in U_{B}$ then $F(h)=s t$. 
For a set $S$ of edges in $G$, let $F(S)$ be the set of edges in $\mathcal{N}$, defined by $F(S)=\{F(h) \mid$ $h \in S \backslash M\}$. The function $F$ is not one-to-one, because the inverse image of an edge $s h$ $(h \in M)$ can be any edge $a h_{B}, a \in U_{A}$.

Clearly, if $M \cup S$ contains an augmenting $M$-alternating path, then $F(S)$ contains an $s-t$ path in $\mathcal{N}$, and vice versa. Let $\mathcal{F}=\left\{F(S) \mid S \in \mathcal{S}_{0}^{c}\right\}$.

Since, by assumption, $m<n,\left|\mathcal{S}_{0}^{c}\right|=2 n-m+k-3 \geqslant m+k-1$. If $N$ is a matching of size $n$, then $M \cup N$ contains an augmenting $M$-alternating path, and hence $F(N)$ contains an $s-t$ path. Hence, by Theorem 24 and Theorem 25, either

(i) there exists an $\mathcal{F}$-rainbow $s-t$ path $P$, or

(ii) $\left|\mathcal{S}_{0}^{c}\right|=m+k-1$ and $\mathcal{F}$ is regimented.

In case (i), as mentioned above, $P$ yields an augmenting $M$-alternating path, whose application yields a larger rainbow matching. So we may assume (ii). Let $\mathcal{R}=(\mathcal{Q}, I)$ be the regimentation of $\mathcal{F}$. Let $F^{-1}(\mathcal{I E}(\mathcal{R}))=\left(S \in \mathcal{S}_{0}^{c} \mid F(S) \in \mathcal{I E}(\mathcal{R})\right)$. Since at most $k-2$ sets $S \in \mathcal{S}$ are empty and $|\mathcal{I E}(\mathcal{R})|=\left|\mathcal{S}_{0}^{c}\right|-|\mathcal{E}(\mathcal{R})|=k-1$ by Lemma $16, \bigcup F^{-1}(\mathcal{I E}(\mathcal{R}))$ is non-empty.

Claim 37. It is possible to choose $M$ so that $\bigcup \mathcal{I E}(\mathcal{R}) \neq \emptyset$.

This means that $\bigcup F^{-1}(\mathcal{I E}(\mathcal{R})) \backslash M \neq \emptyset$.

Proof. Assume, for contradiction, that $\bigcup F^{-1}(\mathcal{I E}(\mathcal{R})) \subseteq M$. Since $\bigcup F^{-1}(\mathcal{I E}(\mathcal{R}))$ is nonempty, there is an element $S_{0} \in \mathcal{S}_{0}$ such that $f_{S_{0}} \in M \cap \bigcup F^{-1}(\mathcal{I E}(\mathcal{R}))$. Let $S_{1}$ be a set in $F^{-1}(\mathcal{I E}(\mathcal{R}))$ containing $f_{S_{0}}$. By the condition of the theorem, $\bigcup F^{-1}(\mathcal{I E}(\mathcal{R})) \cup S_{0}$ contains a matching of size $n$. This, in turn, means that there exists an edge $f \in \bigcup F^{-1}(\mathcal{I E}(\mathcal{R})) \cup$ $S_{0} \backslash M$. Since by assumption $\bigcup F^{-1}(\mathcal{I E}(\mathcal{R})) \subseteq M$, we have $f \in S_{0}$. Now we can consider $\mathcal{S}_{1}=\left(\mathcal{S}_{0} \backslash\left\{S_{0}\right\}\right) \cup\left\{S_{1}\right\}$ as a represented set of $M$ by changing the roles of $S_{0}$ and $S_{1}$. Let $\tilde{\mathcal{F}}=\left(F(S) \mid S \in \mathcal{S}_{1}^{c}\right)$. Then by the same reasoning as above, we may assume that $\tilde{\mathcal{F}}$ is regimented by $\tilde{\mathcal{R}}=(\tilde{\mathcal{Q}}, \tilde{I})$. By Lemma 23 , we have $F\left(S_{0}\right) \in \mathcal{I E}(\tilde{\mathcal{R}})$ and $f \in S_{0} \backslash M$, which implies $\bigcup \mathcal{I E}(\tilde{\mathcal{R}}) \neq \emptyset$.

So, we assume $\bigcup \mathcal{I E}(\mathcal{R}) \neq \emptyset$. Let $p q$ be an edge in $F(S)$ for some $F(S) \in \mathcal{I E}(\mathcal{R})$. By Lemma $18, p q$ is a backward edge on some path $Q \in \mathcal{Q}$. Let $Q=s y_{1} y_{2} \ldots y_{c} t$. For each $1 \leqslant i<c$ let $e_{i}$ be the edge connecting the $\left(y_{i}\right)_{A}$ with $\left(y_{i+1}\right)_{B}$, in $G$ (these are the $F^{-1}$ images of the edges of $Q$ ).

Let $\ell$ be such that $p=y_{\ell}$. As $p$ is an edge in $M, p$ is contained in a set $S_{p} \in \mathcal{S}_{0}$. By the condition of the theorem, the set $S_{p} \cup \bigcup F^{-1}(\mathcal{I E}(\mathcal{R}))$ contains a matching $N$ of size $n$. Since $|M|<n, N$ contains an edge $a x$, where $a \in U_{A}$ (recall that $U_{A}=A \backslash \bigcup M$ ). Suppose $x \in U_{B}$. If $a x \in \bigcup F^{-1}(\mathcal{I E}(\mathcal{R}))$, then $M \cup\{a x\}$ is a rainbow matching, contradicting the maximality of $M$. Thus we have $a x \in S_{p}$. Let $q=y_{\ell^{\prime}}$ for some $\ell^{\prime}<\ell$. Now consider

$$
N=\left(M \cup\left\{a x, p_{A} q_{B}\right\} \cup\left\{\left(y_{i}\right)_{A}\left(y_{i+1}\right)_{B} \mid \ell^{\prime} \leqslant i \leqslant \ell-1\right\}\right) \backslash\left\{y_{\ell^{\prime}}, y_{\ell^{\prime}+1}, \ldots, y_{\ell}\right\} .
$$

Since $p_{A} q_{B} \in S$ and $\left\{\left(y_{i}\right)_{A}\left(y_{i+1}\right)_{B} \mid \ell^{\prime} \leqslant i \leqslant \ell-1\right\}$ has enough represented sets in $I^{-1}(Q)$, then $N$ is a rainbow matching. However, it is a contradiction to the maximality of $M$ since $N$ has size $|M|+1$. 
Hence, we may assume that $x$ lies on an edge $h$ of $M$, meaning that $s h$ is an edge in $F\left(S_{p}\right) \cup \bigcup \mathcal{I E}(\mathcal{R})$. Since all edges in $\bigcup \mathcal{I E}(\mathcal{R})$ are backwards, and $s h$ is not a backward edge on any path, sh belongs to $F\left(S_{p}\right)$.

Let $h \in V\left(Q_{h}\right)$ for $Q_{h} \in \mathcal{Q}$, and let $P$ be the $s-t$ path $s h Q_{h}$. Let $\tilde{P}$ be a path in $F^{-1}(P)$, whose first vertex is $a$, meaning that its first edge belongs to $S_{p}$. Let $X \triangle Y$ be the symmetric difference of $X$ and $Y$, that is, $X \triangle Y=(X \backslash Y) \cup(Y \backslash X)$. Let $N=M \triangle E(\tilde{P})$.

Consider two possibilities:

Possibility I: $h=y_{d}$ for $d \leqslant \ell$.

In this case $N$ is an $\mathcal{S}$-rainbow matching of size $m+1$ : we let the first edge, $a h_{B}$, represents $S_{p}$, and the other edges in $E(\tilde{P}) \backslash M$ has a represented sets in $I^{-1}(Q)$ and keep all other representations as they are. Since the edge in $M$ representing $S_{p}$ is removed by the symmetric difference, this assignment of representation yields an $\mathcal{S}$-rainbow matching.

Possibility II: Either $h \notin V(Q)$ or $h=y_{d}$ for $d>\ell$.

In this case, $N$ is not $\mathcal{S}$-rainbow, since there are two edges representing $S_{p}$, namely $p$ and $a h_{B}$. But this is rectifiable, using the edge $p q$. Suppose that $q=y_{b}$, where $b<\ell$. Let $C$ be the cycle whose edges are $p_{A} q_{B}, q, e_{b}, y_{b+1}, e_{b+1}, \ldots, e_{\ell-1}, p=y_{\ell}$. Let $N^{\prime}=N \triangle E(C)$. Then $N^{\prime}$ is a matching of size $m+1$, and it is $\mathcal{S}$-rainbow, since $S_{p}$ is represented in it just once - by the edge $a h_{B}$.

\section{Somewhere over the rainbow - two possible strengthenings}

It is possible that Theorem 8 can be given a strong cooperation generalisation.

Conjecture 38. Let $\mathcal{F}$ be a family of $2 k-1$ sets of edges in a bipartite graph. If $\nu(\bigcup \mathcal{K}) \geqslant \min (|\mathcal{K}|, k)$ for every $\mathcal{K} \subseteq \mathcal{F}$ then $\nu_{R}(\mathcal{F}) \geqslant k$.

This generalises the following theorem from [2]:

Theorem 39. If $\mathcal{F}=\left(F_{1}, \ldots, F_{2 k-1}\right)$ is a family of matchings in a bipartite graph, and $\left|F_{i}\right|=\min (i, k)$ for all $i$, then there exists an $\mathcal{F}$-rainbow matching of size $k$.

Here is another possible strong version of Theorem 8 .

Conjecture 40. Let $\mathcal{F}=\left(F_{1}, \ldots, F_{2 k-1}\right)$ be a system of bipartite sets of edges, sharing the same bipartition, and suppose that $\nu\left(F_{i}\right) \geqslant k$ for all $i \leqslant 2 k-1$. Let $V^{\prime}$ be a copy of $V$ disjoint from $V$, let $F_{i}^{\prime}$ be a copy of $F_{i}$ on $V^{\prime}(i \leqslant 2 k-1)$ and let $\tilde{F}_{i}=F_{i} \cup F_{i}^{\prime}$ for $i \leqslant 2 k-1$. Then the system $\left(\tilde{F}_{i} \mid i \leqslant 2 k-1\right)$ has a full rainbow matching.

This implies Theorem 2, since by the pigeonhole principle either $V$ or $V^{\prime}$ contains a rainbow matching of size $k$. Conjecture 40 would follow from the following conjecture of the first author and Eli Berger [1].

Conjecture 41. $n$ matchings of size $n$ in any graph have a rainbow matching of size $n-1$. 


\section{Acknowledgements}

This paper is a part of a project that has received funding from the European Union's Horizon 2020 research and innovation programme under the Marie Skllodowska-Curie grant agreement no. 823748. This work was supported by the Russian Federation Government in the framework of MegaGrant no. 075-15-2019-1926 when R. Aharoni worked on Sections 1, 2 and 3 of the paper. The research of R. Aharoni was supported by the Israel Science Foundation (ISF) grant no. 2023464 and the Discount Bank Chair at the Technion. The research of J. Briggs was supported by ISF Grant No. 326/16, ISF Grant No. 1162/15, and ISF Grant No. 409/16. The research of M. Cho was supported by the National Research Foundation of Korea (NRF) grant funded by the Korea government(MSIT) (No. NRF-2020R1F1A1A01048490). The research of J. Kim was supported by BSF Grant No. 2016077, ISF Grant No. 1357/16 and the Institute for Basic Science, Republic of Korea (IBS-R029-C1). This work was completed while J. Briggs and J. Kim were post-doctoral research fellows at the Technion.

The authors thank the anonymous referees for their helpful comments.

\section{References}

[1] R. Aharoni, E. Berger, Rainbow matchings in $r$-partite $r$-graphs, Electron. J. Combin. 16 (2009) \#R119.

[2] R. Aharoni, E. Berger, D. Kotlar and R. Ziv, Degree conditions for matchability in 3-partite hypergraphs, J. Graph Theory 87 (2018), 61-71.

[3] R. Aharoni, R. Holzman, Z. Jiang, Rainbow fractional matchings, Combinatorica 39 (2019) 1191-1202.

[4] R. Aharoni, D. Kotlar, R. Ziv, Uniqueness of the extreme cases in theorems of Drisko and Erdős-Ginzburg-Ziv, European J. Combin., 67 (2018) 222-229.

[5] J. L. Arocha, I. Bárány, J. Bracho, R. Fabila, L. Montejano, Very colorful theorems, Discrete \& Comp. Geom. 42(2) (2009) 142-154.

[6] I. Bárány, A generalization of Carathéodory's theorem, Discrete Math. 40 (1982) $141-152$.

[7] A. A. Drisko, Transversals in row-latin rectangles, J. Combin. Theory, Ser. A 84 (1998) 181-195.

[8] A. F. Holmsen, The intersection of a matroid and an oriented matroid, Adv. Math. 290 (2016) 1-14.

[9] A. F. Holmsen, J. Pach, H. Tverberg, Points surrounding the origin, Combinatorica, 28.6 (2008), 633-644.

[10] A. Holmsen, S. Lee, Leray numbers of complexes of graphs with bounded matching number, arXiv:2003.11270 (2020).

[11] G. Kalai, R. Meshulam, A topological colourful Helly theorem, Adv. Math. 191 (2005) 305-311. 
[12] G. Kalai and R. Meshulam, Intersections of Leray complexes and regularity of monomial ideals, J. Combin. Theory, Ser. A 113 (2006) 1586-1592.

[13] G. Wegner, $d$-Collapsing and nerves of families of convex sets, Arch. Math. 26 (1975) 317-321. 